\title{
Optimal four-dimensional codes over GF(8)
}

\author{
Chris Jones \\ Department of Mathematics and Computer Science \\ St. Mary's College of California \\ Moraga, CA 94575 \\ cjones@stmarys-ca.edu
}

\author{
Angela Matney \\ Department for the Blind and Vision Impaired \\ 397 Azalea Avenue \\ Richmond, VA 23227 \\ angela.matney@dbvi.virginia.gov \\ Harold Ward \\ Department of Mathematics \\ University of Virginia \\ Charlottesville, VA 22904 \\ hnw@virginia.edu
}

Submitted: Oct 21, 2005; Accepted: Jan 17, 2006; Published: Apr 28, 2006

MR Subject Classifications: 94B05, 51E22

\begin{abstract}
We prove the nonexistence of several four-dimensional codes over GF(8) that meet the Griesmer bound. The proofs use geometric methods based on the analysis of the weight structure of subcodes. The specific parameters of the codes ruled out are: $[111,4,96],[110,4,95],[102,4,88],[101,4,87],[93,4,80]$, and the sequence $[29-j, 4,24-j]$, for $j=0,1,2$.
\end{abstract}

\section{Introduction}

An $[n, k, d]_{q}$ code is a linear code of length $n$ and dimension $k$ over the finite field $\mathrm{GF}(q)$, for which the minimum distance between different codewords is $d$. Such a code is traditionally called "optimal" if $n$ is as small as possible among linear codes with the same $k$ and $d$. The famous Griesmer bound asserts that the minimum value $n_{q}(k, d)$ of $n$ satisfies

$$
n \geq g_{q}(k, d)=d+\left\lceil\frac{d}{q}\right\rceil+\ldots+\left\lceil\frac{d}{q^{k-1}}\right\rceil,
$$

and codes meeting this bound are called Griesmer codes. Optimal codes have been the object of research for some time. As with many combinatorial problems dealing with structures meeting bounds, optimal codes often exhibit special properties. These generally relate to the geometrical setting for linear codes that is commonly invoked. The 
important theorem of Belov says that if $q$ and $k$ are fixed, then Griesmer codes exist for large enough $d$. Its proof can be framed in a natural way with the geometric setting.

The two survey articles by Hill [5] and Hill and Kolev [6] present background material and elaborate on the concepts just described. Hirschfeld's comprehensive book [7] contains a nutshell view of the geometric aspect of codes. A web server maintained by Brouwer [2] gives lower and upper bounds on $d$ in terms of $n$ and $k$ for $q=2,3,4,5,7,8,9$, from which a range on $n_{q}(k, d)$ can be inferred. In a paper [11] directly relevant to ours, Maruta presents some ranges for $d$ in terms of general $q$ for which $n_{q}(4, d)=g_{q}(4, d)$ or $g_{q}(4, d)+1$, along with ranges for which it is certain that $n_{q}(4, d)>g_{q}(4, d)$.

In this paper we shall deal with some possible Griesmer codes over GF(8). As might be expected, the larger the field, the more involved the problem. Partly in response, we address certain codes that could exhibit divisibility properties. A linear code is divisible if all of its word weights share a common divisor larger than 1. Optimal codes are often divisible, and this is especially true of Griesmer codes; the paper [13] surveys some of the results in this direction. The advantage of divisibility is evident: the number of possibilities for word weights is diminished and the investigation of the code correspondingly simplified.

Our work aims at showing certain Griesmer codes do not exist. One sad consequence is that the geometric patterns that arise must evaporate with the disappearance of the codes! Perhaps the patterns could be employed in a positive way in another context.

\section{Preliminaries}

Before specializing to the main subject of this paper, four-dimensional codes over $G F(8)$, we shall give some introductory comments and set the geometric stage that will be used.

Let $C$ be a linear code of length $n$ and dimension $k$ over the field $\operatorname{GF}(q)$. The support $\operatorname{supp}(c)$ of a word $c$ in $C$ is the set of coordinate positions at which $c$ has nonzero entries; and the weight $w t(c)$ is $|\operatorname{supp}(c)|$. The support of $C$ itself is the union of the supports of the members of $C$, and the support length $n(C)$ is the size of this support. Code $C$ can be modified in two standard ways: a punctured code arises from deleting a given set $S$ of coordinates from all the codewords (and being mindful of the fact that the resulting code may have lower dimension); and a shortened code is the punctured code of the subcode comprising the words having zeros at the positions in $S$. (These codes are obtained by puncturing or shortening at $S$.) In particular, we have the residual code $\operatorname{Res}(C, c)$ of $C$ at a chosen codeword $c$, the code obtained by puncturing $C$ at $\operatorname{supp}(c)$.

Lemma 1 [4] Let $C$ be an $[n, k, d]_{q}$ code, and let $c$ be a member of $C$. Let $w=w t(c)$ and suppose that $w<q d /(q-1)$. Then $\operatorname{Res}(C, c)$ is an $[n-w, k-1, d-w+\lceil w / q\rceil]_{q}$ code.

This lemma is key in inductive arguments: if no code with the residual parameters exists for a given value of $w$ then there can be no word of weight $w$ in $C$.

The MacWilliams identities are of paramount importance and we use them in the following form: for a code $C$ of length $n$ and dimension $k$ over $\operatorname{GF}(q)$, let $A_{i}$ be the 
number of words of weight $i$ in $C$ and $B_{j}$ the number of words of weight $j$ in the dual $C^{\perp}$ of $C$ (if the code needs specifying, we write things like $A_{i}(C)$ ). Then for $0 \leq m \leq n$,

$$
\sum_{i=m}^{n}\left(\begin{array}{c}
i \\
m
\end{array}\right) A_{i}=q^{k-m} \sum_{j=0}^{m}\left(\begin{array}{c}
n-j \\
m-j
\end{array}\right)(q-1)^{m-j}(-1)^{j} B_{j}
$$

(see, for example, $\left[8\right.$, Section 7.2, equation $\left.\left(\mathrm{M}_{2}\right)\right]$ ). An application of the MacWilliams identities involves the observation that if $b$ is a word in $C^{\perp}$ with $w t(b)=j$, and we shorten $C$ at $\operatorname{supp}(b)$, the resulting code has length $n-j$ but dimension at least $k-j+1$. Consequently, if it is known that no $[n-j, k-j+1, d]_{q}$ code exists, we can conclude that $B_{j}=0$ [5, Lemma 3.3]. If $B_{j}=0$ for the values $j=1, \ldots, m$, then the first $m+1$ MacWilliams identities have right-hand sides expressed by the parameters $n, k, q$ alone. They thus give a collection of equations satisfied by the $A_{i}$ independent of the particular code.

The ray c determined by a nonzero codeword $c$ is the set of nonzero scalar multiples of $c$, that is, the nonzero members of the span of $c$. These multiples all have weight $w t(c)$ and that common weight is declared to be the weight $w t(\mathbf{c})$ of the ray. If $c$ is a nonzero codeword in a ray $\mathbf{c}$, we speak of $\operatorname{Res}(C, c)$ as the residual at $\mathbf{c}$, since $\operatorname{Res}(C, c)$ depends only on $\operatorname{supp}(c)$. We shall often refer to rays simply by their weights: "a 92-ray" or just "a 92" means a ray of weight 92. Rays can be construed as the points of the projective space $\mathbf{C}$ determined by $C$. We shall adopt a geometric language in what follows, except that "ray" will be used in place of "point." In general, the projective set that comprises the rays in a subspace $D$ of $C$ will be denoted by the matching boldface symbol, $\mathbf{D}$. We set $a_{i}=A_{i} /(q-1)$ and $b_{j}=B_{j} /(q-1)$; these are the numbers of rays of weight $i$ in $C$ and $j$ in $C^{\perp}$, respectively, and we refer to the $a_{i}$ as forming the weight distribution of $\mathbf{C}$ itself. The MacWilliams identities can be divided by $q-1$ to give corresponding identities connecting the $a_{i}$ and the $b_{j}$ (on making allowance for $A_{0}=B_{0}=1$ ):

$$
\begin{aligned}
\sum_{i=1}^{n} a_{i} & =\frac{q^{k}-1}{q-1} \\
\sum_{i=m}^{n}\left(\begin{array}{c}
i \\
m
\end{array}\right) a_{i} & =q^{k-m}\left\{\left(\begin{array}{c}
n \\
m
\end{array}\right)(q-1)^{m-1}+\sum_{j=1}^{m}\left(\begin{array}{c}
n-j \\
m-j
\end{array}\right)(q-1)^{m-j}(-1)^{j} b_{j}\right\}
\end{aligned}
$$

with $m>0$ in the second line. The case $m=1$ is singled out as the Average Weight Equation $(A W E)$. Here $b_{1}$ is the number of coordinate positions at which all words in $C$ show zeros. Traditionally one sets $b_{1}=z$, making $n(C)=n-z$. Then $A W E$ reads:

$$
\sum_{i=1}^{n} i a_{i}=q^{k-1}(n-z)=q^{k-1} n(C) .
$$

Suppose that $C$ is an $[n, k, d]_{q}$ code with $b_{1}=b_{2}=0$, as will be the case for the main codes to be discussed. Define the displacement $\delta(\mathbf{c})$ of a ray $\mathbf{c}$ by $\delta(\mathbf{c})=w t(\mathbf{c})-d$. 
Then for given $\alpha$ and $\beta$, the first three of the MacWilliams identities can be combined to produce the quadratic relation

$$
\begin{aligned}
\sum_{\delta=0}^{n-d}(\delta & -\alpha)(\delta-\beta) a_{\delta+d}=\frac{q^{k}-1}{q-1} \alpha \beta-\left\{q^{k-1} n-d \frac{q^{k}-1}{q-1}\right\}(\alpha+\beta) \\
& +q^{k-2} n((q-1) n+1)-2 d q^{k-1} n \\
& +d^{2} \frac{q^{k}-1}{q-1}
\end{aligned}
$$

If we set $Q(\delta)=(\delta-\alpha)(\delta-\beta)$, the summation is $\sum_{\mathbf{c}} Q(\delta(\mathbf{c}))$, taken over $\mathbf{C}$. We shall denote the right side by $Q(\mathbf{C})$.

From here on, we shall take $q=8$ and omit the subscript " 8 " on the code parameters. We need the weight distributions of some potential residual codes, all of them Griesmer. They are readily obtained from the MacWilliams identities and are tabulated here:

$$
\begin{array}{cc}
\text { Code parameters } & \text { Weight Distribution } \\
{[10,3,8]} & a_{8}=45, a_{10}=28 \\
{[9,3,7]} & a_{7}=36, a_{8}=9, a_{9}=28 \\
{[8,3,6]} & a_{6}=28, a_{7}=16, a_{8}=29 \\
{[7,3,5]} & a_{5}=21, a_{6}=21, a_{7}=31 \\
{[6,3,4]} & a_{4}=15, a_{5}=24, a_{6}=34 \\
{[5,3,3]} & a_{3}=10, a_{4}=25, a_{5}=38 \\
{[4,3,2]} & a_{2}=6, a_{3}=24, a_{4}=43 \\
{[3,3,1]} & a_{1}=3, a_{2}=21, a_{3}=49
\end{array}
$$

This result on divisibility will be applied frequently:

Lemma 2 [13, Proposition 13] If $C$ is a Griesmer code over $\mathrm{GF}(8)$ whose minimum weight is a multiple of 8 , then $C$ is an even code: all of its word weights are divisible by 2.

The quadratic relation (1) will often be used in conjunction with an analysis of ray weights for a line. Suppose that the nine rays $\mathbf{c}_{i}$ of a line $\mathbf{L}$ in an $[n, k, d]$ code have displacements $\delta_{i}=\delta\left(\mathbf{c}_{i}\right)$, ray $\mathbf{c}_{0}$ being singled out. Then by $A W E$,

$$
\delta_{0}+d+\sum_{i=1}^{8}\left(\delta_{i}+d\right)=8(n-z)
$$

where $z=z(\mathbf{L})$ is the number of coordinate positions at which all nine rays show 0s. Thus

$$
\sum_{i=1}^{8} \delta_{i}=8 n-9 d-\delta_{0}-8 z
$$

This relation serves to restrict the possibilities for the values of the $\delta_{i}$. Notice that $\mathbf{L}$ projects onto a ray of weight $n-d-\delta_{0}-z$ in the residual at $\mathbf{c}_{0}$. 


\section{Two non-existent Griesmer codes}

The preceding results provide the initial steps of the investigation of the codes to be dealt with in the paper. Here is an algorithm to be followed for an $[n, 4, d]$ code:

\section{Algorithm 3}

1. From implied residual parameters, eliminate selected values as potential codeword weights. Let $\Delta$ be the displacement set, the set of allowed displacements remaining after this step.

2. Rule out further members of $\Delta$ one at a time by taking one as $\delta_{0}$ and showing that equation (3) cannot be satisfied with the $\delta_{i}$ coming from $\Delta$.

3. Having trimmed $\Delta$ as far as possible and having shown that $b_{1}=b_{2}=0$, apply the quadratic relation (1) for well-chosen $\alpha$ and $\beta$ either to arrive at a contradiction or to obtain further restrictions on the weight enumerator.

In presenting the analysis for a particular code, we may skimp on details.

\subsection{On the $[111,4,96]$ code}

Suppose that $C$ is a $[111,4,96]$ code. Since $C$ is a Griesmer code and the minimum weight is a multiple of 8 , Lemma 2 implies that $C$ is an even code. Following the algorithm we have:

1. For the code $C, a_{i}=0$ for $i=98,100,106$, and 108: by Lemma 1 , the residuals for words of these weights have parameters $[13,3,11],[11,3,9],[5,3,4]$, and $[3,3,2]$, and all of these are ruled out by the Griesmer bound. The displacement set is now $\Delta=\{0,6,8,14\}$.

2. We have $a_{102}=a_{110}=0$ for the code $C$ : equation (3) becomes

$$
\sum_{i=1}^{8} \delta_{i}=8 \times 111-9 \times 96-\delta_{0}-8 z=24-\delta_{0}-8 z .
$$

For a ray $\mathbf{c}$ of weight 102 , there must be a line with $z=2$ containing $\mathbf{c}$, namely the preimage of a ray of weight 7 in the residual at c. But $\delta_{0}=6$ and $z=2$ requires $\sum_{i=1}^{8} \delta_{i}=2$, which is not feasible with the $\delta_{i}$ in $\Delta$. Thus $a_{102}=0$ and the displacement set shrinks to $\{0,8,14\}$. Similarly, a line with $z=1$ on a ray of weight 110 also requires $\sum_{i=1}^{8} \delta_{i}=2$, still not possible.

3. At this point the displacement set is just $\{0,8\}$. The Griesmer bound prohibits $[110,4,96]$ and $[109,3,96]$ codes, so $b_{1}=b_{2}=0$. The quadratic relation (1) applies with $\alpha=0$ and $\beta=8$ to give

$$
\sum_{\delta=0,8} \delta(\delta-8) a_{\delta+96}=1152
$$


But the left side is 0 , and we have a contradiction. Thus:

Theorem 4 There is no $[111,4,96]$ code.

\subsection{On the $[102,4,88]$ code}

A $[102,4,88]$ code is Griesmer and even, by Lemma 2. The Griesmer bound rules out $[101,4,88]$ and $[100,3,88]$ codes, so that $b_{1}=b_{2}=0$.

1. In a $[102,4,88]$ code, $a_{90}=a_{98}=a_{100}=0$ : the needed residual codes with parameters $[12,3,10],[4,3,3]$ and $[2,3,1]$ do not exist. Thus $\Delta=\{0,4,6,8,14\}$.

2. Further, $a_{94}=a_{102}=0$. For a ray of weight 94 on a line with $z=2$ required by the $[8,3,6]$ residual, (3) reads $\sum_{i=1}^{8} \delta_{i}=2$, not allowed by $\Delta$. Likewise, a line on a ray of weight 102 , necessarily with $z=0$, requires $\sum_{i=1}^{8} \delta_{i}=10$, but now using $\Delta=\{0,4,8,14\}$. Again, this is not possible.

3. Our displacement set is now $\{0,4,8\}$, and we use the quadratic relation (1) with $\alpha=0, \beta=8$ to see that $(4-0)(4-8) a_{92}=-16 a_{92}=384$, which cannot be. Hence

Theorem 5 No [102, 4,88] code exists.

\section{Corresponding punctured codes}

We next show that there are no $[110,4,95]$ or $[101,4,87]$ codes, using a modification of step 2 of Algorithm 3. The quadratic relation (1) with $\alpha=\beta=0$ becomes the square relation

$$
\sum_{\mathbf{c}} \delta(\mathbf{c})^{2}=q^{k-2} n((q-1) n+1)-2 d q^{k-1} n+d^{2} \frac{q^{k}-1}{q-1}=S(\mathbf{C}) .
$$

With $q=8$ and $k=4$, we have

$$
S(\mathbf{C})=64 n(7 n+1)-1024 n d+585 d^{2} .
$$

Let $\mathbf{c}_{0}$ be a fixed ray with $\delta\left(\mathbf{c}_{0}\right)=\delta_{0}$, and for a line $\mathbf{L}$ on $\mathbf{c}_{0}$ put $S(\mathbf{L})=\sum_{\mathbf{c} \in \mathbf{L}-\left\{\mathbf{c}_{0}\right\}} \delta(\mathbf{c})^{2}$. Then $\sum_{\mathbf{L}} S(\mathbf{L})=S(\mathbf{C})-\delta_{0}^{2}$, the sum over the lines $\mathbf{L}$ containing $\mathbf{c}_{0}$. We sort these lines by the corresponding values of $z$. When the residual at $\mathbf{c}_{0}$ is three-dimensional and $a_{j}^{\prime}$ is the number of rays of weight $j$ in it, there are $a_{n-d-\delta_{0}-z}^{\prime}$ such lines (lower dimensional cases will be dealt with separately). If $\delta_{0}$ and $\delta_{1}, \ldots, \delta_{8}$ are the displacements of the rays on a line $\mathbf{L}$, and $z=z(\mathbf{L})$, the $\delta_{i}$ are related by $(3): \sum_{i=1}^{8} \delta_{i}=8 n-9 d-\delta_{0}-8 z$. Let $S_{z}$ be the maximum of $\sum_{i=1}^{8} \delta_{i}^{2}$ subject to this relation, with the $\delta_{i}$ coming from the current displacement set. Then $\sum_{\mathbf{L}} S(\mathbf{L}) \leq \sum_{j} a_{j}^{\prime} S_{n-d-\delta_{0}-j}$. If this last sum falls short of $S(\mathbf{C})-\delta_{0}^{2}$ in the square relation, then we have a contradiction, and no ray of displacement $\delta_{0}$ exists. 
For reference, the inequality needing to be established is

$$
\sum_{j} a_{j}^{\prime} S_{n-d-\delta_{0}-j}<S(\mathbf{C})-\delta_{0}^{2}=64 n(7 n+1)-1024 d n+585 d^{2}-\delta_{0}^{2}
$$

(again with modifications for residuals of dimension smaller than 3).

Examination of the partitions $\sum_{i=1}^{8} \delta_{i}=8 n-9 d-\delta_{0}-8 z$ for the maximum of $\sum_{i=1}^{8} \delta_{i}^{2}$ is expedited by the fact that if $\delta_{i} \geq \delta_{j}$ and $\varepsilon>0$, then $\left(\delta_{i}+\varepsilon\right)^{2}+\left(\delta_{j}-\varepsilon\right)^{2}>\delta_{i}^{2}+\delta_{j}^{2}$. There will generally be only a few "extremal" partitions in which one cannot move to another legitimate one (the $\delta_{i}$ in the displacement set) having higher $\sum_{i=1}^{8} \delta_{i}^{2}$ using one or more changes of pairs from $\delta_{i}, \delta_{j}$ to $\delta_{i}+\varepsilon, \delta_{j}-\varepsilon$. From these, the one with largest $\sum_{i=1}^{8} \delta_{i}^{2}$ is then $S_{z}$.

\subsection{On the $[110,4,95]$ code}

For a $[110,4,95]$ code, $b_{1}=b_{2}=0$ as for the others. Equation (3) becomes

$$
\sum_{i=1}^{8} \delta_{i}=8 \times 110-9 \times 95-\delta_{0}-8 z=25-\delta_{0}-8 z .
$$

We have $S(\mathbf{C})=6665$ in (5).

1. The Griesmer bound applied to residual parameters rules out rays of weight 97,98 , 99, 105, 106, 107, and 108 in C. The displacement set is then

$$
\Delta=\{0,1,5,6,7,8,9,14,15\} .
$$

2. In this step, we rule out weights 110, 109, and 104.

110: Here $\delta_{0}=15$, and $z$ can only be 0 . Then $\sum_{i=1}^{8} \delta_{i}=10$. The extremal partition is $9+1+6 \times 0$, and $S_{0}=82$. As $73 \times 82=5986<6665-15^{2}=6440$, weight 110 is ruled out.

109: Now $\delta_{0}=14$ and $\Delta=\{0,1,5,6,7,8,9,14\}$. The residual is one-dimensional, so there are 64 lines with $z=0$ and 9 with $z=1$ on a ray of weight 109 . The equation for $z=0$ is $\sum_{i=1}^{8} \delta_{i}=11$, with extremal partitions $9+2 \times 1+5 \times 0$ and $6+5+6 \times 0$, making $S_{0}=83$. At $z=1$, we just need $\sum_{i=1}^{8} \delta_{i}=3$; the only partition is $3 \times 1+5 \times 0$, and $S_{1}=3$. The comparison for (6) is

$$
64 \times 83+9 \times 3=5339<6665-14^{2}=6469 .
$$

This inequality rules out 109 .

104: $\Delta=\{0,1,5,6,7,8,9\}$ and $\delta_{0}=9$. For the $[6,3,4]$ residual, $a_{4}=15, a_{5}=24$, and $a_{6}=34$. When $z=0$, we need $\sum_{i=1}^{8} \delta_{i}=16$. The extremal partition is $9+7+6 \times 0$, giving $S_{0}=130$. At $z=1, \sum_{i=1}^{8} \delta_{i}=8$, the extremal partition is 
$8+7 \times 0$, and $S_{1}=64$. Finally, for $z=2$, the partition is $8 \times 0$, making $S_{2}=0$. Our inequality is

$$
34 \times 130+24 \times 64=5956<6665-9^{2}=6584,
$$

which eliminates 104.

3. The displacement set is now $\Delta=\{0,1,5,6,7,8\}$. This time put $Q=(\delta-4)^{2}$, making $Q(\mathbf{C})=10065$. The largest value of $\delta^{2}$ in $\Delta$ is 64 , so that $\sum_{\mathbf{c}} Q(\mathbf{c}) \leq$ $585 \times 16=9360$. As that is too small, we have arrived at

Theorem 6 There is no $[110,4,85]$ code.

\subsection{On the $[101,4,87]$ code}

As ever, $b_{1}=b_{2}=0$ for a $[101,4,87]$ code. The line equation (3) still requires

$$
\sum_{i=1}^{8} \delta_{i}=8 \times 101-9 \times 87-\delta_{0}-8 z=25-\delta_{0}-8 z ;
$$

and $S(\mathbf{C})=6489$.

1. The residual step in Algorithm 3 eliminates the weights 89, 90, 97, 98, and 99, making $\Delta=\{0,1,4,5,6,7,8,9,13,14\}$.

2. We eliminate weights step-by-step again:

101: For $z=0$, the only case, $\sum_{i=1}^{8} \delta_{i}=11$; the two extremal partitions are $9+1+1+5 \times 0$ and $7+4+6 \times 0$, so that $S_{0}=83$. With $\delta_{0}=14$, the comparison (6) is just

$$
73 \times 83=6059<6489-14^{2}=6293
$$

and 101 is eliminated.

100: Here $\delta_{0}=13$. As before, $z=0$ for 64 lines; we need $\sum_{i=1}^{8} \delta_{i}=12$, and the two extremal partitions are $9+3 \times 1+4 \times 0$ and $8+4+6 \times 0$. Thus $S_{0}=84$. For the remaining nine lines with $z=1, \sum_{i=1}^{8} \delta_{i}=4$, for which we have $4+7 \times 0$ and $S_{1}=16$. Once again, we have a contradiction:

$$
64 \times 84+9 \times 16=5520<6489-13^{2}=6320 .
$$

From here on, we work up through lower weights. The details for the various weights are much the same, and we shall simply tabulate values.

91: $\delta_{0}=4$ and $\Delta=\{0,1,4,5,6,7,8,9\}$.

$$
\begin{aligned}
& z \text { \# lines } \sum_{i=1}^{8} \delta_{i}=\text { extremal partitions } S_{z} \\
& 028 \quad 21 \quad\left\{\begin{array}{cc}
2 \times 9+3 \times 1+3 \times 0 \\
9+8+4+5 \times 0
\end{array}\right. \\
& 10 \\
& \begin{array}{lllll}
2 & 45 & 5 & 5+7 \times 0 & 25
\end{array}
\end{aligned}
$$


The eliminating inequality reads

$$
28 \times 165+45 \times 25=5745<6489-4^{2}=6473 .
$$

92: $\delta_{0}=5$ and $\Delta=\{0,1,5,6,7,8,9\}$.

\begin{tabular}{|c|c|c|c|c|}
\hline & \# lines & $\sum_{i=1}^{8} \delta_{i}=$ & extremal partitions & $S_{z}$ \\
\hline & 28 & 20 & $2 \times 9+2 \times 1+4 \times 0$ & 164 \\
\hline & 9 & 12 & $\begin{array}{c}9+3 \times 1+4 \times 0 \\
7+5+6 \times 0\end{array}$ & \\
\hline & 36 & 4 & $4 \times 1+4 \times 0$ & \\
\hline
\end{tabular}

Eliminating inequality:

$$
28 \times 164+9 \times 84+36 \times 4=5492<6489-5^{2}=6464 .
$$

93: $\delta_{0}=6$ and $\Delta=\{0,1,6,7,8,9\}$.

$\begin{array}{ccccc}z & \# \text { lines } & \sum_{i=1}^{8} \delta_{i}= & \text { extremal partitions } & S_{z} \\ 0 & 29 & 19 & 2 \times 9+1+5 \times 0 & 163 \\ 1 & 16 & 11 & 9+2 \times 1+5 \times 0 & 83 \\ 2 & 28 & 3 & 3 \times 1+5 \times 0 & 3\end{array}$

Eliminating inequality:

$$
29 \times 163+16 \times 83+28 \times 3=6139<6489-6^{2}=6453 .
$$

At this point, no further eliminations succeed.

3. The displacement set is now $\{0,1,7,8,9\}$, and the weight enumerator, with $a_{95}$ and $a_{96}$ as parameters, is

$$
\begin{aligned}
& a_{87}=\frac{4048}{7}-a_{95}-\frac{16}{7} a_{96} \\
& a_{88}=-\frac{385}{3}+\frac{4}{3} a_{95}+3 a_{96} \\
& a_{94}=\frac{2836}{21}-\frac{4}{3} a_{95}-\frac{12}{7} a_{96}
\end{aligned}
$$

Integrality implies that $a_{96} \neq 0$. For the residual of a 96, $a_{5}^{\prime}=38$. With $\delta_{0}=$ $96-87=9$, the line equation is $\sum_{i=1}^{8} \delta_{i}=16-8 z$, so that only for $z=0$ can there be a 9 among the $\delta_{i}$, and then at most one. This all means that $0<a_{96} \leq 38+1=39$. 
To rule out the code, we could apply an extension theorem of Maruta [12, Theorem 1.5] to provide an additional restriction on the $a_{i}$. Since the code does not extend to a $[102,4,88]$ code (because that doesn't exist!), Maruta's theorem requires that

$$
\sum_{i \neq 87} A_{i}=A_{88}+A_{94}+A_{96} \geq 8^{4-2}(2 \times 8-1)-1=959 .
$$

From the weight enumerator, this inequality is $47+16 a_{96} \geq 959$, or $a_{96} \geq 57$-an inconsistency.

However, here is a "stand-alone" proof drawing on the circle of ideas in [13] that involves the same computation as in Maruta's theorem: if $\lambda_{1}, \ldots, \lambda_{101}$ are the coordinate functionals of the code, then for a codeword $c, w t(c) \equiv \sum \lambda_{i}(c)^{7}(\bmod 2)$. When the hypothetical code $C$ is viewed as a space over $\mathrm{GF}(2)$, the sum on the right is a polynomial function of degree at most 3; so too is the function $c \rightarrow 1+w t(c)(\bmod 2)$. As the GF $(2)$ dimension of $C$ is 12 , this function defines a word $w$ in the Reed-Muller code $\mathcal{R}(3,12)$ (see, for example, $\left[9\right.$, Chapters 13, 15]). The weight $w t(w)$ of $w$ is $1+A_{88}+A_{94}+A_{96}=48+16 a_{96}$. Since $a_{96} \leq 39, w t(w) \leq 672$. The minimum weight of $\mathcal{R}(3,12)$ is $2^{12-3}=512$, so that $512 \leq w t(w)<2 \times 512$; in particular, $48+16 a_{96} \geq 512$ and $a_{96} \geq 29$. By the theorem of Kasami and Tokura [9, Chapter 15, Theorem 11], wt $(w)$ then has the form 1024(1-2 $\left.2^{-h}\right)$. Now $1024\left(1-2^{-h}\right) \leq 672$ forces $h=1$, and $w$ is a word of minimum weight in $\mathcal{R}(3,12)$. Consequently $w$ is the characteristic function of a 9-flat in $C$ as a $\operatorname{GF}(2)$-space [9, Chapter 13, Theorem 5]; the flat is actually a subspace, because the zero word is in it. But since $w t(\alpha c)=w t(c)$ for $\alpha \in \mathrm{GF}(8)$, this subspace is a $\mathrm{GF}(8)$-subspace of $C$. That is: the words of even weight in $C$ form a 3-dimensional subcode of $C$.

Thus we see a $[101,3,88]$ code with nonzero word weights $88,94,96$. It is a Griesmer code and for it, $b_{1}=0$. The MacWilliams identities give $a_{88}=\frac{199}{3}+\frac{1}{3} a_{96}, a_{94}=\frac{20}{3}-\frac{4}{3} a_{96}$. But then $a_{96} \leq 5$, incompatible with $a_{96} \geq 29$.

Theorem 7 No [101, 4,87] code exists.

\section{On the code sequence $[29-j, 4,24-j]_{8}, j=0,1,2$}

We include-or rather, exclude-these three codes for the record. They would all be Griesmer and would form a sequence of codes obtained by successive puncturings. Thus if the lowest one, [27, 4, 22], does not exist, none of them do. For a hypothetical [27, 4, 22] code, equation (3) at $z=2$ for a ray of weight 25 reads

$$
\sum \delta_{i}=8 \times 27-9 \times 22-3-2 \times 8=-1,
$$

so that $A_{25}=0$ (the residual dimension is only 2 , and a line with $z=2$ is required). As the three successive shortenings $[26,4,22],[25,3,22]$, and $[24,2,22]$ all violate the Griesmer bound, $B_{1}=B_{2}=B_{3}=0$, by Lemma 1 . But then the MacWilliams identities give the disconcerting result that $A_{23}=-1008-5 A_{27}$; so the code does not exist. 


\section{On the $[93,4,80]_{8}$ code}

\subsection{Initial results}

For the rest of the discussion, let $C$ be a hypothetical $[93,4,80]$ code, with corresponding projective space C. As will become apparent, a more intricate analysis is needed now. Such a code would meet the Griesmer bound, so that by [13, Proposition 13] the weights of the codewords in $C$ are multiples of 2 . Residuals rule out all weights except 80, 84, 86,88 , and 92 , making the displacement set $\Delta=\{0,4,6,8,12\}$. If there were an 86-ray, $\delta_{0}=6$, the preimage of a 5-ray from the residual would be a line on the 86-ray with $z=2$. The line equation (3) would read

$$
\sum_{i=1}^{8} \delta_{i}=8 n-9 d-\delta_{0}-8 z=24-6-16=2
$$

and that cannot be completed from the members of $\Delta$.

Again the Griesmer bound prohibits $[92,4,80]$ and $[91,3,80]$ codes, so that $b_{1}=b_{2}=0$. Then the MacWilliams identities give the following weight distribution, with $a_{92}$ as a parameter:

$$
a_{80}=471-a_{92}, a_{84}=24+3 a_{92}, a_{88}=90-3 a_{92}, b_{3}=982-8 a_{92} .
$$

\subsection{Lines}

As with the previous codes, we use an analysis of the lines, but in rather more detail. The displacement set is $\Delta=\{0,4,8,12\}$, and the line equation is

$$
\sum_{i=0}^{8} \delta_{i}=24-8 z
$$

Let the type of line $\mathbf{L}$ be the sequence $\mathbf{a}_{0} \mathbf{a}_{4} \mathbf{a}_{8} \mathbf{a}_{12}$, where $\mathbf{a}_{\delta}$ is the number of rays $\mathbf{c}$ on $\mathbf{L}$ with $\delta(\mathbf{c})=\delta$. We refer to $\mathbf{L}$ as an $\mathbf{a}_{0} \mathbf{a}_{4} \mathbf{a}_{8} \mathbf{a}_{12}$-line, or as a $z(\mathbf{L})$-line if we don't need the specific type. Then the possibilities for the types are:

$\begin{array}{cccc}\text { type } & z(\mathbf{L}) & \text { type } & z(\mathbf{L}) \\ \mathbf{9 0 0 0} & 3 & \mathbf{3 6 0 0} & 0 \\ \mathbf{7 2 0 0} & 2 & \mathbf{4 4 1 0} & 0 \\ \mathbf{8 0 1 0} & 2 & \mathbf{5 2 2 0} & 0 \\ \mathbf{5 4 0 0} & 1 & \mathbf{5 3 0 1} & 0 \\ \mathbf{6 2 1 0} & 1 & \mathbf{6 0 3 0} & 0 \\ \mathbf{7 0 2 0} & 1 & \mathbf{6 1 1 1} & 0 \\ \mathbf{7 1 0 1} & 1 & \mathbf{7 0 0 2} & 0\end{array}$

Let $l_{a b c d}$ be the number of abcd-lines in $\mathbf{C}$. Then we get a set of equations by counting rays and pairs of rays by their weights, according as to how many lines they lie in. There 
are

$$
\frac{\left(8^{4}-1\right)\left(8^{4}-8\right)}{\left(8^{2}-1\right)\left(8^{2}-8\right)}=4745
$$

lines altogether, and each ray is in $\left(8^{3}-1\right) /(8-1)=73$ lines; each pair of distinct rays is in one line. For example,

$$
\begin{aligned}
73 a_{88} & =l_{8010}+l_{6210}+2 l_{7020}+l_{4410}+2 l_{5220}+3 l_{6030}+l_{6111} \\
a_{84} a_{88} & =2 l_{6210}+4 l_{4410}+4 l_{5220}+l_{6111} \\
\left(\begin{array}{c}
a_{92} \\
2
\end{array}\right) & =l_{7002}
\end{aligned}
$$

Two more equations come from the weight distributions of the residual codes in (2): the preimage of a 7-ray in an $\mathbf{8 4}$ residual must be a 7200-line. Thus each $\mathbf{8 4}$ is on 36 such lines. As each line is counted twice by its $\mathbf{8 4} \mathrm{s}, l_{7200}=18 a_{84}$. Similarly, $l_{8010}=10 a_{88}$. The whole set of equations can be solved with $a_{92}, l_{6030}$, and $l_{7020}$ as parameters; but we shall only need a few of the results:

$$
\begin{aligned}
& l_{5400}=-1071+42 a_{92}+l_{7020} \\
& l_{4410}=-6 a_{92}^{2}+333 a_{92}-4590+3 l_{6030}+2 l_{7020} \\
& l_{3600}=2 a_{92}^{2}-121 a_{92}+1837-l_{6030}-l_{7020}
\end{aligned}
$$

We know from $0 \leq a_{88}=90-3 a_{92}$ that $a_{92} \leq 30$ to begin with. As

$$
0 \leq 3 l_{3600}+l_{4410}+l_{5400}=12 a_{92}-150
$$

we infer that $a_{92} \geq 13$. Similarly,

$$
0 \leq l_{5400}+l_{3600}+l_{6030}=2 a_{92}^{2}-79 a_{92}+766 \approx 2\left(a_{92}-17.09\right)\left(a_{92}-22.41\right) .
$$

Thus we obtain

Condition 8 Either $13 \leq a_{92} \leq 17$ or $a_{92} \geq 23$.

\subsection{Planes}

To refine the results, we consider subcodes $P$ of $C$ of dimension 3 , corresponding to (projective) planes $\mathbf{P}$ in $\mathbf{C}$.

\subsubsection{Short planes}

First suppose that $P$ is a shortening of $C$, necessarily a $[92,3,80]$ code by the Griesmer bound. The plane $\mathbf{P}$ will be called a short plane. Since 7002 -lines have $z=0$, a short plane $\mathbf{P}$ contains no such line and $a_{92}(\mathbf{P})=0$ or 1 . In what follows, we shall use the parameters $e=a_{88}(\mathbf{P})$ and $f=a_{92}(\mathbf{P})$ when dealing with planes. Solving the 
MacWilliams equations with $B_{1}(P)=0$ gives $a_{80}(\mathbf{P})=61+e+2 f, a_{84}(\mathbf{P})=12-2 e-3 f$. With the restriction $f=0,1$, these distributions result:

$\begin{array}{lllllllll}a_{80}(\mathbf{P})= & 63 & 67 & 66 & 65 & 64 & 63 & 62 & 61 \\ a_{84}(\mathbf{P})= & 9 & 0 & 2 & 4 & 6 & 8 & 10 & 12 \\ a_{88}(\mathbf{P})= & 0 & 6 & 5 & 4 & 3 & 2 & 1 & 0 \\ a_{92}(\mathbf{P})= & 1 & 0 & 0 & 0 & 0 & 0 & 0 & 0\end{array}$

Now do line counting in $\mathbf{P}$. There are just seven possibilities for line types, those with

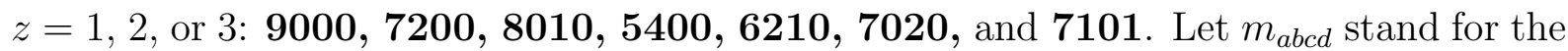
number of abcd-lines in $\mathbf{P}$, and create the same sorts of equations as for the $l_{a b c d}$. This time there are 73 lines and each ray is in 9 . When $f=1$, there is just the one solution, given in the table below. For $f=0$, the solution of the equations is

$$
\begin{aligned}
& m_{9000}=2 e+22 \\
& m_{7200}=8(6-e) \\
& m_{8010}=4 e \\
& m_{5400}=\frac{1}{2}(e-1)(e-6) \\
& m_{6210}=e(6-e) \\
& m_{7020}=\frac{1}{2} e(e-1) \\
& m_{7101}=0
\end{aligned}
$$

Then $m_{7200} \geq 0$ implies that $e \leq 6$; but $0 \leq m_{5400}=(e-1)(e-6) / 2$ shows that if $e<6$, then in fact $e=0$ or 1 . Thus there are only four possibilities for the weight distribution of $\mathbf{P}$ and the corresponding $m_{a b c d}$. The type of each plane will be taken as the type abcd of 1-line that the plane contains, and we shall refer to the plane as an abcd short plane.

$\begin{array}{ccccc}\text { number of planes }= & s_{5400} & s_{6210} & s_{7020} & s_{7101} \\ a_{80}= & 61 & 62 & 67 & 63 \\ a_{84}= & 12 & 10 & 0 & 9 \\ e= & 0 & 1 & 6 & 0 \\ f= & 0 & 0 & 0 & 1 \\ m_{9000}= & 22 & 24 & 34 & 28 \\ m_{7200}= & 48 & 40 & 0 & 36 \\ m_{8010}= & 0 & 4 & 24 & 0 \\ m_{5400}= & 3 & 0 & 0 & 0 \\ m_{6210}= & 0 & 5 & 0 & 0 \\ m_{7020}= & 0 & 0 & 15 & 0 \\ m_{7101}= & 0 & 0 & 0 & 9\end{array}$

Codes with each of these parameters exist, although they may not all appear in $C$. Each 1-line occurs in exactly one short plane, and it follows that $l_{5400}=3 s_{5400}, l_{6210}=5 s_{6210}$, $l_{7020}=15 s_{7020}$, and $l_{7101}=9 s_{7101}$; of course $s_{7101}=a_{92}$. The new ingredient is the divisibilities of the $l$-values. In particular $l_{7020}$ has to be a multiple of 15 with

$$
-42 a_{92}+1071 \leq l_{7020} \leq 2 a_{92}^{2}-121 a_{92}+1837
$$


by the nonnegativity of the $l_{a b c d}$ in (8). When $a_{92}=17$ this reads $357 \leq l_{7020} \leq 358$, with no room for a multiple of 15 ; consequently

Condition 9 For the hypothetical code $C, a_{92}=17$ is ruled out.

Counting all rays in $\mathbf{C}$, we obtain these equations for the $s_{a b c d}$, observing that a ray of weight $w$ appears in $93-w$ of the short planes:

$$
\begin{aligned}
& 93=s_{5400}+s_{6210}+s_{7020}+s_{7101} \\
& 13 a_{80}=61 s_{5400}+62 s_{6210}+67 s_{7020}+63 s_{7101} \\
& 9 a_{84}=12 s_{5400}+10 s_{6210}+9 s_{7101} \\
& 5 a_{88}=s_{6210}+6 s_{7020} \\
& a_{92}=s_{7101}
\end{aligned}
$$

Their solution is

$$
\begin{aligned}
& s_{5400}=5 s_{7020}+14 a_{92}-357 \\
& s_{6210}=-6 s_{7020}-15 a_{92}+450 \\
& s_{7101}=a_{92}
\end{aligned}
$$

Substituting $l_{7020}=15 s_{7020}$ into $(8)$ gives a fresh parameterization:

$$
\begin{aligned}
& l_{5400}=42 a_{92}+15 s_{7020}-1071 \\
& l_{4410}=-6 a_{92}^{2}+333 a_{92}+30 s_{7020}+3 l_{6030}-4590 \\
& l_{3600}=2 a_{92}^{2}-121 a_{92}+1837-15 s_{7020}-l_{6030}
\end{aligned}
$$

\subsubsection{Long planes}

Consider now long planes, three-dimensional subcodes $P$ with $z=0$, and do sort of line analysis as for the short planes. On solving the MacWilliams identities with $n=93$ and $b_{1}=0$, again with $a_{88}(\mathbf{P})=e$ and $a_{92}(\mathbf{P})=f$, we obtain the values $a_{80}(\mathbf{P})=45+e+2 f$ and $a_{84}(\mathbf{P})=28-2 e-3 f$. All line types are possible in the equations giving numbers of rays and ray pairs in terms of line counts. From the solution we retain two key equations:

$$
\begin{aligned}
& m_{5301}=f(9-e-f) \\
& m_{5400}=\frac{1}{2}(9-e-f)(14-e-3 f)-3 m_{3600}-m_{4410}
\end{aligned}
$$

The nonnegativity of $m_{5301}$ and $m_{5400}$ then imply that $f(9-e-f) \geq 0$ and $(9-e-$ $f)(14-e-3 f) \geq 0$. In addition, $a_{84}(\mathbf{P}) \geq 0$ gives $2 e+3 f \leq 28$. These inequalities produce the following bounds:

Lemma 10 Let $e=a_{88}(\mathbf{P})$ and $f=a_{92}(\mathbf{P})$, for a long plane $\mathbf{P}$. If $f=0$, then either $0 \leq e \leq 9$ or $e=14$. If $e=0$, then either $f=9$ or $0 \leq f \leq 4$. Moreover, $e+f \leq 9$ except when $e=14$. 
Lemma 11 Let $\mathbf{c}$ be a $\mathbf{9 2}$ in $\mathbf{C}$. Then the numbers of lines containing $\mathbf{c}$ are these:

$$
\begin{array}{ccccc}
\text { line type: } & \mathbf{7 1 0 1} & \mathbf{5 3 0 1} & \mathbf{6 1 1 1} & \mathbf{7 0 0 2} \\
\text { number containing } \mathbf{c :} & 9 & 2 a_{92}-25 & 90-3 a_{92} & a_{92}-1
\end{array}
$$

The short plane containing $\mathbf{c}$ is a 7101-plane and it contains all nine 7101-lines on $\mathbf{c}$.

Proof. Ray $\mathbf{c}$ is on 73 lines, necessarily of the four types tabulated. The 7002-lines are the lines joining $\mathbf{c}$ to the $a_{92}-1$ other $92 \mathrm{~s}$, and the 6111 -lines join $\mathbf{c}$ to the $88 \mathrm{~s}$. Thus if there are $x$ 7101-lines and $y$ 5301-lines on $\mathbf{c}$, then

$$
\begin{aligned}
x+y & =73-\left(a_{92}-1\right)-a_{88}=2 a_{92}-16 \\
x+3 y & =a_{84}-a_{88}=6 a_{92}-66,
\end{aligned}
$$

whence the totals. The statement about the short plane follows from the compositions in (10).

The following result avoids later nuisances:

Lemma 12 For the code $C, a_{92} \leq 29$.

Proof. Suppose that $a_{92}=30$, the case to be ruled out. Then $a_{88}=90-3 a_{92}=0$, so that long planes have $e=0$ and either $f=9$ or $0 \leq f \leq 4$, by Lemma 10. Picture a 7002-line and the nine planes on it, all of them long; we might speak informally of "fanning" the 7002-line. Outside the 7002-line, the nine planes contain 28 of the $\mathbf{9 2 \mathrm { s }}$ among them. Suppose that $x$ of the planes have $f=9$. By the possibilities for $f$, it follows that $7 x+2(9-x) \geq 28$, or $x \geq 2$. Thus each 7002 -line is on at least two planes with $f=9$. Each such plane contains $\left(\begin{array}{l}9 \\ 2\end{array}\right)=36$ of the 7002-lines. If we take one of the planes with $f=9$, we see at least 36 more covering the 7002-lines in it; so there are at least 37 planes with $f=9$. Two of these meet in at most one 7002-line, so there are at least $37 \times 36-\left(\begin{array}{c}37 \\ 2\end{array}\right)=666$ 7002-lines (the partial sums in the inclusion-exclusion formula alternately over-estimate and under-estimate, the import of the Bonferroni inequalities [3, Section 4.7]). But from the line type list (7), the only lines containing two 92s are 7002-lines. Thus $l_{7002}=\left(\begin{array}{c}30 \\ 2\end{array}\right)=435<666$, a beastly inconsistency.

At this point we have

Condition 13 For the hypothetical code $C, 13 \leq a_{92} \leq 16$ or $23 \leq a_{92} \leq 29$,

by Condition 8, Condition 9, and Lemma 12 .

\subsubsection{Octoplanes}

The $[9,3,7]$ residual at an $\mathbf{8 4}$ contains nine words of weight 8 , and they are the nonzero words of a two-dimensional subcode. The preimage of this in the projective space $\mathbf{C}$ of our hypothetical $[93,4,80]$ code is a long plane containing the $\mathbf{8 4}$ that will be called the octoplane of the $\mathbf{8 4}$; the $\mathbf{8 4}$ is an octoray for the plane. Each $\mathbf{8 4}$ is an octoray for just one octoplane, but it is conceivable that a plane is the octoplane for more than one $\mathbf{8 4}$. 
If $\mathbf{p}$ is an $\mathbf{8 4}$ contained in a line $\mathbf{L}$, the image of $\mathbf{L}$ in the residual code of $\mathbf{p}$ has weight $9-z(\mathbf{L})$. Thus the 1-lines containing $\mathbf{p}$ are the nine lines containing $\mathbf{p}$ in the octoplane of $\mathbf{p}$. On the other hand, if $\mathbf{p}$ is in a long plane $\mathbf{P}$ that is not the octoplane of $\mathbf{p}$, then the image of $\mathbf{P}$ in the residual at $\mathbf{p}$ is a two-dimensional code containing four rays of weight 7 , one of weight 8 , and four of weight 9 (the only other possible ray weight distribution for such a subcode). Examination of the line types (7) yields:

Lemma 14 Let $\mathbf{p}$ be an $\mathbf{8 4}$ in a long plane $\mathbf{P}$. If $\mathbf{P}$ is the octoplane of $\mathbf{p}$, then all the

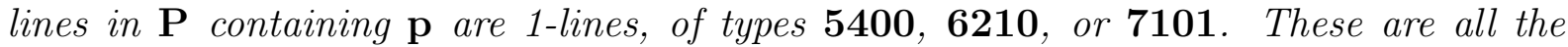
1-lines of $\mathbf{C}$ that contain $\mathbf{p}$. But if $\mathbf{p}$ is not an octoray for $\mathbf{P}$, then of the lines in $\mathbf{P}$ that contain $\mathbf{p}$, four are $\mathbf{7 2 0 0 - l i n e s ; ~ o n e ~ i s ~ a ~ 5 4 0 0 - l i n e , ~ a ~ 6 2 1 0 - l i n e , ~ o r ~ a ~ 7 1 0 1 - l i n e ; ~ a n d ~ f o u r ~}$ have types from the list 3600, 4410, 5220, 5301, 6111.

Proposition 15 There is exactly one octoray for an octoplane.

Proof. Let $\mathbf{O}$ be an octoplane and suppose $\mathbf{p}$ and $\mathbf{q}$ are two distinct octorays for $\mathbf{O}$. Then the line pq is either a $\mathbf{6 2 1 0}$-line or a $\mathbf{5 4 0 0}$-line. There cannot be a $\mathbf{9 2}$ in $\mathbf{O}$. For if $\mathbf{t}$ is such a ray, then pt and $\mathbf{q t}$ are two 7101-lines through $\mathbf{t}$. By Lemma 11, pt and $\mathbf{q t}$ determine the short plane on $\mathbf{t}$, not a long plane like $\mathbf{O}$.

All the $\mathbf{8 4}$ s on $\mathbf{O}$ must be octorays for $\mathbf{O}$. To see that, suppose that $\mathbf{r}$ is an $\mathbf{8 4}$ not on pq. Then pr and $\mathbf{q r}$ are two 1-lines through $\mathbf{r}$, and $\mathbf{r}$ is necessarily an octoray for $\mathbf{O}$, by Lemma 14. Moreover, if $\mathbf{s}$ is a further $\mathbf{8 4} \mathbf{~ o n ~} \mathbf{~ p q}$, then $\mathbf{s}$ is also an octoray by virtue of ps $(=\mathrm{qs})$ and $\mathbf{r s}$. If there is no $\mathbf{8 4}$ in $\mathbf{O}$ outside $\mathbf{p q}$, then $\mathrm{O}$ cannot contain a $\mathbf{7 2 0 0}$-line, as one of its 84 s would not be on pq. Thus any further 84 on pq is not on a 7200 -line, and so it must be an octoray for $\mathbf{O}$.

It follows that $O$ contains no line of type $7200,3600,4410,5220$, or 5301 , and, as we said, no 92. Now augment all the long plane line equations (used at the beginning of this subsection) by those declaring these counts to be 0 , and solve. With $e=a_{88}(\mathbf{O})$, as usual, and $m_{6030}$ for a parameter, we find

$$
\begin{aligned}
m_{9000} & =10-2 e+2 m_{6030} \\
m_{8010} & =3 m_{6030}-4 e \\
m_{7020} & =\frac{1}{2} e(e-1)-3 m_{6030} \\
m_{6210} & =e(14-e) \\
m_{5400} & =\frac{1}{2}(e-9)(e-14)
\end{aligned}
$$

and all other line counts equal to 0 . From $m_{7020} \geq 0$ and $m_{8010} \geq 0$ we get

$$
4 e \leq 3 m_{6030} \leq \frac{1}{2} e(e-1),
$$

making $e(e-9) \geq 0$. Then Lemma 10 imply that $e=0,9$, or 14 . But with $f=0$ and $e=14$ we have $a_{84}(\mathbf{O})=28-2 e-3 f=0$, which is not consistent with $\mathbf{O}$ 's being an octoplane. Thus $e=0$ or 9 . 
First suppose that $e=0$. Then $m_{6030}=0$ also, and the counts for lines in $\mathbf{O}$ are just $m_{9000}=10$ and $m_{5400}=63$. As each $\mathbf{5 4 0 0}$-line is on a $\mathbf{5 4 0 0}$ short plane, we have $63 \leq s_{5400}=5 s_{7020}+14 a_{92}-357$; we also have $0 \leq-6 s_{7020}-15 a_{92}+450$, both inequalities by (11). Eliminating $s_{7020}$, we get $a_{92} \geq 30$. Thus, in fact, $a_{92}=30$; but that possibility has been ruled out in Proposition 12.

If $e=9$, then (14) becomes $36 \leq 3 m_{6030} \leq 36$, making $m_{6030}=12$ and $m_{7020}=0$. Hence each 88 in $\mathrm{O}$ is on four of the $\mathbf{6 0 3 0}$-lines. The nine $\mathbf{8 8 s}$ and the twelve $\mathbf{6 0 3 0}$-lines now form an affine plane of order 3. But by [1, Theorems 5.2 and 6.1], that plane does not embed in our projective plane of order 8.

Corollary 16 Each 1-line is on as many octoplanes as the number of $\mathbf{8 4} s$ on the line.

Proof. The octoplane of each 84 on the line contains the line, by Lemma 14, and by the proposition the octoplanes for different $\mathbf{8 4}$ s are distinct.

Corollary 17 The 92-lines of an octoplane are either 7020-lines or the lines through the octoray.

Proof. If a 1-line $\mathbf{L}$ is not a $\mathbf{7 0 2 0}$-line, it contains an $\mathbf{8 4}$, say $\mathbf{p}$. If $\mathbf{p}$ is not the octoray, $\mathbf{p}$ is on a 1-line $\mathbf{M}$ through the octoray. Then if $\mathbf{L} \neq \mathbf{M}$, ray $\mathbf{p}$ is on the two 1-lines $\mathbf{L}$ and $\mathbf{M}$ and so an octoray for the plane, by Lemma 14. But now the octoplane has two octorays, contradicting the proposition.

\subsection{The inequality $a_{92}<23$}

The key result for the rest of the discussion is a consequence of the facts on octoplanes.

Proposition 18 An 88 in $\mathbf{C}$ is on at most two 6210 short planes.

Proof. Suppose that $\mathbf{p}$ is an $\mathbf{8 8}$ on three type $\mathbf{6 2 1 0}$ short planes, $\mathbf{S}_{1}, \mathbf{S}_{2}$, and $\mathbf{S}_{3}$. Then $\mathbf{p}$ is the only $\mathbf{8 8}$ in each of them, by the table (10). Since the lines containing an $\mathbf{8 8}$ in these planes have types $\mathbf{8 0 1 0}$ and $\mathbf{6 2 1 0}$, with $z=2$ and $z=1$, respectively, the intersections $\mathbf{S}_{i} \cap \mathbf{S}_{j}$ of different planes are $\mathbf{8 0 1 0}$-lines. The five $\mathbf{6 2 1 0}$-lines in $\mathbf{S}_{1}$ are on ten octoplanes, by Corollary 16 . These must meet $\mathbf{S}_{2}$ and $\mathbf{S}_{3}$ in $\mathbf{8 0 1 0}$-lines, because an 88 in an octoplane is on just one $\mathbf{6 2 1 0}$-line in it - namely, the line joining the $\mathbf{8 8}$ to the octoray - by Corollary 17. At most five of the octoplanes can meet $\mathbf{S}_{2}$ and $\mathbf{S}_{3}$ in $\mathbf{S}_{2} \cap \mathbf{S}_{3}$, one from each 6210-line in $\mathbf{S}_{1}$; otherwise, two octoplanes would contain $\mathbf{S}_{2} \cap \mathbf{S}_{3}$ and the same 6210-line and so coincide. None of the octoplanes contains $\mathbf{S}_{1} \cap \mathbf{S}_{2}$ or $\mathbf{S}_{1} \cap \mathbf{S}_{3}$ (since none is $\mathbf{S}_{1}$ itself); so the five or more not containing $\mathbf{S}_{2} \cap \mathbf{S}_{3}$ meet $\mathbf{S}_{2}$ in one of its two 8010-lines different from $\mathbf{S}_{1} \cap \mathbf{S}_{2}$ and $\mathbf{S}_{2} \cap \mathbf{S}_{3}$. Likewise, these octoplanes meet $\mathbf{S}_{3}$ in one of two 8010-lines. But there can only be four such planes, one for each pairing of an 8010-line from $S_{2}$ with an 8010-line from $\mathbf{S}_{3}$.

This result implies that $a_{92}<23$. 
Proposition 19 For the hypothetical code $C$, it must be that $a_{92}<23$. Thus $13 \leq a_{92} \leq$ 16.

Proof. By the proposition, each 88 is on at most two 6210 short planes; such planes contain just one 88. Thus $s_{6210} \leq 2 a_{88}$. From (11), this becomes $6 s_{7020} \geq 270-9 a_{92}$. On the other hand, with

$$
l_{3600}=2 a_{92}^{2}-121 a_{92}+1837-15 s_{7020}-l_{6030}
$$

from $(12), l_{3600} \geq 0$ gives $15 s_{7020} \leq 2 a_{92}^{2}-121 a_{92}+1837$. Sandwiching $s_{7020}$ leads to another quadratic inequality:

$$
0 \leq 2 a_{92}^{2}-\frac{197}{2} a_{92}+1162 \approx 2\left(a_{92}-19.59\right)\left(a_{92}-29.66\right) .
$$

Hence $a_{92} \leq 19$ or $a_{92} \geq 30$. By the established restrictions on $a_{92}$ in Condition 13 , it follows that $13 \leq a_{92} \leq 16$.

\subsection{Ruling out the code}

As a preliminary for the final step, we need an analogue of Lemma 11:

Lemma $\mathbf{2 0}$ Let $\mathbf{p}$ be an $\mathbf{8 8}$ in $\mathbf{C}$. Then we have the following counts for the lines on $\mathbf{p}$ :

$\begin{array}{cccccccc}\text { line type } & \mathbf{8 0 1 0} & \mathbf{6 2 1 0} & \mathbf{7 0 2 0} & \mathbf{4 4 1 0} & \mathbf{5 2 2 0} & \mathbf{6 0 3 0} & \mathbf{6 1 1 1} \\ \text { count } & 10 & 5 s_{6} & 5 s_{7} & x_{1} & x_{2} & x_{3} & a_{92}\end{array}$

Here $s_{6}$ is the number of $\mathbf{6 2 1 0}$ short planes that $\mathbf{p}$ is on and $s_{7}$ the number of $\mathbf{7 0 2 0}$ short planes containing $\mathbf{p}$. We have the relations

$$
\begin{aligned}
& s_{7}=5-s_{6} \\
& x_{1}=2 a_{92}-26-5 s_{6}+x_{3} \\
& x_{2}=64-3 a_{92}+5 s_{6}-2 x_{3} .
\end{aligned}
$$

Proof. Ray $\mathbf{p}$ is on $93-88=5$ short planes of types $\mathbf{6 2 1 0}$ and $\mathbf{7 0 2 0}$. By the data in (10) and the values in (7) and (2), $\mathbf{p}$ is on four 8010-lines and five lines with $z=1$ in each short plane. This gives the first three counts and the $s_{i}$ relation. The count $a_{92}$ for 6111-lines just reflects the lines connecting $\mathbf{p}$ to the $92 \mathrm{~s}$. For the $x_{j}$, we have that the total number of lines is 73 , and the total number of $88 \mathrm{~s}$ appearing is $a_{88}=90-3 a_{92}$. Thus

$$
\begin{aligned}
10+25+x_{1}+x_{2}+x_{3}+a_{92} & =73 \\
a_{88}-1 & =89-3 a_{92}=5 s_{7}+x_{2}+2 x_{3} .
\end{aligned}
$$

The solutions, parameterized by $s_{6}$ and $x_{3}$, are as listed. 
We recall the three values in (12):

$$
\begin{aligned}
& l_{5400}=42 a_{92}+15 s_{7020}-1071 \\
& l_{4410}=-6 a_{92}^{2}+333 a_{92}+30 s_{7020}+3 l_{6030}-4590 \\
& l_{3600}=2 a_{92}^{2}-121 a_{92}+1837-15 s_{7020}-l_{6030} .
\end{aligned}
$$

The nonnegativity of the $l_{a b c d}$ implies that

$$
\begin{aligned}
15 s_{7020} & \geq 1071-42 a_{92} \\
15 s_{7020}+l_{6030} & \leq 2 a_{92}^{2}-121 a_{92}+1837 \\
15 s_{7020}+\frac{3}{2} l_{6030} & \geq 3 a_{92}^{2}-\frac{333}{2} a_{92}+2295 .
\end{aligned}
$$

These inequalities then lead to the following parameter values or ranges:

$\begin{array}{cccccc}a_{92}= & 13 & 14 & 15 & 15 & 16 \\ s_{7020}= & 35 & 33 & 31 & 30 & 27 \\ l_{6030}= & 75 \text { to } 77 & 38 \text { to } 40 & 5 \text { to } 7 & 15 \text { to } 22 & 0 \text { to } 8 \\ a_{88}+a_{92}= & 64 & 62 & 60 & 60 & 58 \\ s_{6210}= & 45 & 42 & 39 & 45 & 48\end{array}$

Now fan a 6030-line: outside the line there are $a_{88}+a_{92}-388 \mathrm{~s}$ and $92 \mathrm{~s}$ on the nine planes (all long) through the 6030-line. As $a_{88}+a_{92}-3 \geq 55$ in all the cases, and 55/9 $>6$, at least one of these planes has $e+f>9$. But such a plane can only be a 14-plane, that is, one with $e=14$ and $f=0$, by Lemma 10 . Thus the 6030 -line must be on at least one 14-plane.

Lemma 21 Let $\mathbf{F}$ be a 14-plane. Then $a_{80}(\mathbf{F})=59$ and $a_{84}(\mathbf{F})=0$. The line counts are parameterized by $m_{6030}$ and they are:

$$
\begin{array}{ccccc}
\text { line type } & \mathbf{9 0 0 0} & \mathbf{8 0 1 0} & \mathbf{7 0 2 0} & \mathbf{6 0 3 0} \\
\text { number } & 38-m_{6030} & 3 m_{6030}-56 & 91-3 m_{6030} & m_{6030}
\end{array}
$$

Here $19 \leq m_{6030} \leq 30$, and all other line counts are 0. Moreover, an $\mathbf{8 8}$ in $\mathbf{F}$ is on four, five, or six 6030-lines.

Proof. The line types listed are the only ones possible for $\mathbf{F}$. As for the more general

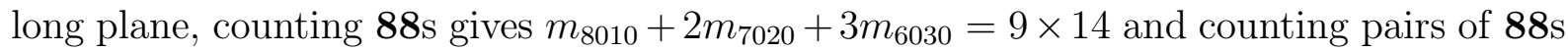
gives $91=m_{7020}+3 m_{6030}$; from this one gets all the line counts. If an individual 88 is on $y_{1}$ 8010-lines, $y_{2}$ 7020-lines, and $y_{3}$ 6030-lines, then $y_{1}+y_{2}+y_{3}=9$ and $y_{2}+2 y_{3}=13$ (the count of 88s). Thus $y_{1}=y_{3}-4$ and $y_{2}=13-2 y_{3}$, from which the limits $4 \leq y_{3} \leq 6$ follow.

Nonnegativity also implies that $19 \leq m_{6030} \leq 30$. (The planes correspond to $[14,3,11]_{8}$ codes by the $\mathbf{8 8} \mathrm{s}$, and these codes have been classified by Marcugini, Milani, and Pambianco [10]. Their results show that $m_{6030} \leq 26$, in fact.) 
With these preliminaries we deal with the four remaining values for $a_{92}$ :

$\boldsymbol{a}_{92}=13$ : In this case, each $\mathbf{6 0 3 0}$-line is actually on at least two 14-planes: since $a_{88}+$ $a_{92}=64$, the eight planes other than a 14-plane on a 6030 -line contain $64-14=5088 \mathrm{~s}$ and 92s not on the 6030-line. As before, $50 / 8+3=9.25$ shows that at least one of the eight planes has $e+f>9$ and so is a 14-plane.

There are at most four 14-planes: if there were a set of five, they would contain at least $5 \times 19-\left(\begin{array}{l}5 \\ 2\end{array}\right)=85$ 6030-lines, more than allowed by (15). On the other hand, if we take one 14-plane, then each of the $\mathbf{6 0 3 0}$-lines on it is in another 14-plane. Thus one would see at least $1+19=2014$-planes.

$\boldsymbol{a}_{92}=14$ : Here $38 \leq l_{6030} \leq 40$, from (15), so there must be just two 14-planes, $\mathbf{F}_{1}, \mathbf{F}_{2}$. As $a_{88}=48$, there are at least $20 \mathbf{8 8}$ s not on them. If $\mathbf{p}$ is one of these $\mathbf{8 8} \mathbf{s}$, then $\mathbf{p}$ is not on a 6030-line. By Lemma 20, $x_{1}=2-5 s_{6}$ for $\mathbf{p}$, so that $s_{6}=0$; that is, $\mathbf{p}$ is not on a 6210 short plane. Similarly, if $\mathbf{p}$ is an $\mathbf{8 8}$ on just one of the $F_{i}$, then $x_{3} \leq 6$, by Lemma 21 , and $0 \leq x_{1} \leq 8-5 s_{6}$, so that $\mathbf{p}$ is on at most one $\mathbf{6 2 1 0}$ short plane. Finally, if $\mathbf{p}$ is an $\mathbf{8 8}$ on $\mathbf{F}_{1} \cap \mathbf{F}_{2}$, then $x_{3} \leq 12$, and $x_{1} \leq 14-5 s_{6}$ implies that $\mathbf{p}$ is on at most two $\mathbf{6 2 1 0}$ short planes.

If $\mathbf{F}_{1} \cap \mathbf{F}_{2}$ contains $q \mathbf{8 8 \mathbf { s }}$, then $q \leq 3$ (maximized when $\mathbf{F}_{1} \cap \mathbf{F}_{2}$ is a $\mathbf{6 0 3 0}$-line). It follows that

$$
s_{6210} \leq(28-q) \times 1+q \times 2=28+q \leq 31
$$

however, $s_{6210}=42$, by $(15)$.

$\boldsymbol{a}_{92}=15$ : The argument is similar to that for $a_{92}=14$. This time $5 \leq l_{6030} \leq 22$, from

(15); so there is one 14-plane, $\mathbf{F}$. For an 88 not in $\mathbf{F}, x_{1}=4-5 s_{6}$, making $s_{6}=0$; the 88 is on no 6210 short plane. An 88 in $\mathbf{F}$ has $x_{3} \leq 6$, so that $x_{1} \leq 10-5 s_{1}$ and the 88 is on at most two $\mathbf{6 2 1 0}$ short planes. Once again the count of $\mathbf{6 2 1 0}$ short planes falls short: it is at most $14 \times 2=28$. However, $s_{6210} \geq 39$.

$\boldsymbol{a}_{92}=16$ : Now $l_{6030} \leq 8$ by (15), and Lemma 21 implies that there are no 14-planes at all: $l_{6030}=0$. Then for all 88s, $x_{1}=6-5 s_{6}$, making $s_{6} \leq 1$. Thus $s_{6210} \leq a_{88}=42$ and yet $s_{6210}=48$, from $(15)$.

We have arrived at our theorem:

Theorem 22 There is no $[93,4,80]_{8}$ Griesmer code.

\section{Acknowledgment}

We wish to than the referee for a careful reading of the manuscript.

\section{References}

[1] M. S. Abdul-Elah, M. W Al-Dhahir, and D. Jungnickel, $8_{3}$ in PG(2, q), Arch. Math. (Basel) 49 (1987), no. 2, 141-150. 
[2] A. E. Brouwer, Linear code bounds, web server located at http://www.win.tue.nl/ aeb/voorlincod.html

[3] L. Comtet, Advanced Combinatorics, D. Reidel, Dordrecht, 1974.

[4] S. Dodunekov, Minimal block length of a linear $q$-ary code with specified dimension and code distance, Problems Inform. Transmission 20 (1984), no. 4, 239-249.

[5] R. Hill, Optimal linear codes, Optimal linear codes. Cryptography and coding, II (Cirencester, 1989), Oxford University Press, New York (1992), 75-104.

[6] R. Hill and E. Kolev, A survey of recent results on optimal linear codes, Combinatorial designs and their applications (Milton Keynes, 1997), Chapman \& Hall/CRC Res. Notes Math. 403, Chapman \& Hall/CRC, Boca Raton, FL (1999), 127-152.

[7] J. W. P. Hirschfeld, Projective Geometries over Finite Fields, 2nd Ed., Oxford University Press, New York, 1998.

[8] W. C. Huffman and V. Pless, Fundamentals of Error-Correcting Codes, Cambridge University Press, Cambridge, 2003.

[9] F. J. MacWilliams and N. J. A. Sloane, The Theory of Error-Correcting Codes, North-Holland Publishing Company, Amsterdam, 1977.

[10] S. Marcugini, A. Milani, and F. Pambianco, Classification of the $[n, 3, n-3]_{q}$ NMDS codes over GF(7), GF(8) and GF(9), Ars Combin. 61 (2001), 263-269.

[11] T. Maruta, On the minimum length of $q$-ary linear codes of dimension four, Discrete Math. 208/209 (1999), 427-435.

[12] T. Maruta, Extendability of linear codes over $\operatorname{GF}(q)$ with minimum distance $d$, $\operatorname{gcd}(d, q)=1$. Discrete Math. 266, nos. 1-3 (2003), 377-385.

[13] Harold N. Ward, Divisible codes-a survey. Serdica Math. J. 27 (2001), no. 4, 263-278. 